\title{
Promotion of Lymphatic Integrity by Angiopoietin-1/ Tie2 Signaling during Inflammation
}

\author{
Kentaro Kajiya, ${ }^{*}$ Hiroyasu Kidoya ${ }^{\dagger}{ }^{\text {Mika Sawane, }}{ }^{*}$ \\ Yuuko Matsumoto-Okazaki, ${ }^{*}$ Haruyo Yamanishi, ${ }^{*}$ \\ Mikio Furuse, ${ }^{\ddagger}$ and Nobuyuki Takakura ${ }^{\dagger}$ \\ From the Shiseido Innovative Science Research Center," \\ Yokohama; the Department of Signal Transduction, ${ }^{\dagger}$ Research \\ Institute of Microbial Diseases, Osaka University, Osaka; and the \\ Department of Physiology and Cell Biology, ${ }^{\ddagger}$ Kobe University, \\ Kobe, Japan
}

The cutaneous lymphatic system plays a major role in tissue fluid homeostasis and inflammation of the skin. Although several lymphangiogenic factors are known to be involved in the formation of lymphatic vessels, the molecular mechanisms that maintain lymphatic integrity and control the functional drainage of interstitial fluid and resolution of inflammation remain unknown. Here we show that angiopoietin-1 (Ang1) enhances lymphatic integrity and function during inflammation. Ang1 transgenic mice under the control of keratin-14 (K14-Ang1) showed attenuated edema formation and inflammation after UV B (UVB) exposure. After UVB irradiation, blood vascular permeability was inhibited in K14-Ang1 mice compared with wild-type (WT) mice. Moreover, lymphatic vessels of WT mice were markedly enlarged and leaky in inflamed skin, whereas K14-Ang1 mice showed relatively contracted lymphatic vessels together with enhanced lymphatic vascularization. Expression of endothelial-specific tight junction molecules claudin-5 and zonula occludens protein 1 (ZO-1) was strongly down-regulated in the inflamed lymphatic vessels of UVB-exposed WT mice, whereas down-regulation of both claudin- 5 and ZO-1 was blocked in UVB-exposed K14-Ang1 mice. In vitro studies revealed that the stability of lymphatic endothelial cells was enhanced in the presence of Ang1, presumably via up-regulation of claudin-5, as well as ZO-1. Claudin-5 knockdown markedly increased the permeability of lymphatic endothelial cells. Overall, our data strongly support the idea that Ang1/Tie2 signaling promotes lymphatic integrity by modulating tight junction molecule expression during inflammation. (AmJ Pathol 2012, 180:1273-1282; DOI: 10.1016/j.ajpath.2011.11.008)
The lymphatic vascular system, which is composed of a dense network of thin-walled capillaries in peripheral tissues such as skin, plays a major role in the maintenance of tissue fluid homeostasis, and its impairment leads to lymphedema. ${ }^{1,2}$ Another important aspect of lymphatic function is the afferent phase of immune response. Because lymphatic vessels are the conduit not only for excess water and macromolecules, but also for inflammatory cells such as macrophages, a detailed understanding of lymphatic function could lead to the development of methods to improve the resolution of inflammation. Indeed, recent findings in mouse models have indicated that the lymphatic vasculature is actively involved in inflammatory processes. ${ }^{2-4}$

Several lymphangiogenic factors, including vascular endothelial growth factor (VEGF)-C/-D/-A, platelet-derived growth factor-BB, angiopoietins, and hepatocyte growth factor (HGF), are known. ${ }^{1,5} \mathrm{It}$ has been shown that inflammation is attenuated in VEGF-C transgenic mice, or by subcutaneous delivery of VEGF-C, as a result of skin lymphangiogenesis, which promotes lymphatic function. ${ }^{4,6}$ In contrast, adenoviral or transgenic delivery of VEGF-A induced the appearance of giant and functionally abnormal lymphatic vessels. ${ }^{7}$ Systematic blockade of VEGF-A markedly reduced cutaneous photosensitivity and inflammation through inhibition of the enlargement of lymphatic vessels. ${ }^{8}$ We also found that leaky and hyperpermeable inflamed lymphatics were associated with upregulation of VEGF-A as well as down-regulation of VEGF-C. ${ }^{6,8}$ Together, these data suggest that lymphangiogenic factors are actively involved in inflammation by mediating structural alteration of the lymphatics. Thus, application of other lymphangiogenic factors, which may directly regulate and/or cooperatively induce functional lymphangiogenesis in inflamed tissue, may have potential as a new strategy to accelerate the resolution of inflammation.

It recently was established that angiopoietin-1 (Ang1) promotes lymphatic vessel formation. ${ }^{9,10}$ However, in

Accepted for publication November 14, 2011.

K.K. and H.K. contributed equally to this work.

Address reprint requests to Kentaro Kajiya, Ph.D., Shiseido Innovative Science Research Center, 2-12-1 Fukuura Kanazawa-ku, Yokohama 2368643, Japan. E-mail: kentaro.kajiya@to.shiseido.co.jp. 
contrast to the detailed understanding of the role of Ang1/ Tie2 signaling in migration, permeability, and inflammation of blood vascular endothelial cells, ${ }^{11}$ it remains unknown how lymphatic function is regulated by Ang1/Tie2 signaling. Structurally, lymphatic endothelial cells possess finely arranged, but unusual and discontinuous, junctions, consisting of adherens junctions, endothelial adhesion molecules, and tight junction proteins (TJPs) such as claudins, which allow the recirculation of fluid and cells transported to peripheral tissues via blood vessels. ${ }^{12}$ It has been reported that claudin-5 knockout mice have increased permeability of the blood-brain barrier, ${ }^{13}$ but the role of claudin-5 in lymphatic function is completely unknown. Here, we investigated how lymphatic function is modulated in angiopoietin-1 transgenic mice under the control of keratin 14 (K14-Ang1), to obtain insight into the molecular regulation of inflammation. Our results indicate that it may be feasible to treat edema and inflammation by the induction of functional lymphangiogenesis with Ang1.

\section{Materials and Methods}

\section{Animals}

Ten female WT mice and $10 \mathrm{~K} 14$-Ang1 transgenic mice ${ }^{14}$ (kindly provided by Dr. George Yanconpoulous) at 8 to 15 weeks of age were exposed to a single dose of 200 $\mathrm{mJ} / \mathrm{cm}^{2}$ UV B (UVB) irradiation, using 10 Toshiba FL-20 SE fluorescent lamps (Toshiba, Tokyo, Japan) that deliver energy in the UVB wavelength range ( 280 to $340 \mathrm{~nm}$, with a maximum at $305 \mathrm{~nm}) .{ }^{15}$ Ten female WT and $10 \mathrm{~K} 14$ Ang1 mice without UVB irradiation also were used as controls. The thickness of the ears was measured every day until day 3 after irradiation. Three days after the UVB irradiation, the ears were removed, embedded in OCT compound, and processed for histologic analyses. All procedures, including measurement of ear thickness and UVB irradiation, were performed under anesthesia. All animal studies were approved by the Shiseido Research Center Committee on Research Animal Care.

\section{Plasma Extravasation and Intravital Lymphangiography}

To determine the blood vascular permeability, a Miles assay was performed as previously described. ${ }^{16}$ Briefly, mice were anesthetized and intravenously injected with $150 \mu \mathrm{L}$ of a $1 \%$ solution of Evans Blue dye in $0.9 \% \mathrm{NaCl}$. At 40 minutes after the dye injection, pictures of the ears were taken and the ears were removed. The dye was eluted from the dissected samples with formamide at $56^{\circ} \mathrm{C}$, and the optical density was measured by spectrophotometry (Biotrak II; GE Healthcare, Fairfield, CT) at $620 \mathrm{~nm}$. An intravital lymphatic permeability assay was conducted as described. ${ }^{8}$ A $1-\mu \mathrm{L}$ aliquot of a $1 \%$ solution of Evans Blue dye was injected intradermally at the inner surface of the rim of the ear, using a 10- $\mu \mathrm{L}$ Hamilton syringe, to visualize lymphatic vessels. The ear was photographed at 1, 5, and 10 minutes after dye injection.

\section{Immunostaining}

Immunofluorescence analysis was performed on 6- $\mu \mathrm{m}$ cryostat sections of mouse skins, using rat monoclonal antibodies against mouse LYVE-1 (MBL, Nagoya, Japan), against mouse Ki-67 antigen (DAKO Cytomation, Glostrup, Denmark), against mouse CD11b (BD Bioscience, Bedford, CA), and against mouse panendothelial cell antigen (clone: MECA-32; BD Bioscience), a hamster monoclonal antibody against mouse podoplanin (Angiobio, Del Mar, CA), and polyclonal antibodies against mouse LYVE-1 (RELIA Tech, Wolfenbuttel, Germany), against human claudin-5 (Santa Cruz Biotechnology, Santa Cruz, CA), against human zonula occludens protein 1 (ZO-1) (Invitrogen, Carlsbad, CA), and against mouse Prox1 (Covance, Emeryville, CA). Corresponding secondary antibodies labeled with AlexaFluor488 or AlexaFluor594 (Molecular Probes, Eugene, OR) were used. Routine H\&E staining also was performed. Sections were examined with an Olympus AX80T microscope (Olympus, Tokyo, Japan) and images were captured with a DP controller digital camera (Olympus). Wholeear skin was stained with monoclonal antibodies against CD31 (clone: MEC-13.3; BD Bioscience) and against podoplanin (Angiobio) and a polyclonal antibody against claudin-5 (Santa Cruz Biotechnology). Confocal microscopic examination of whole skin was performed using a LSM5 (Carl Zeiss, Thornwood, NY), and images were processed further using IMARIS (Carl Zeiss). Morphometric analyses were performed using IP-LAB software (Snanalytics, Fairfax, VA) as described. ${ }^{17}$ Three different fields of each section were examined and the number of vessels per square micrometer, the average vessel size, and the relative tissue area occupied by lymphatic vessels were determined in an area of the dermis within a 200- $\mu \mathrm{m}$ distance from the epidermal-dermal junction. The unpaired Student's t-test was used to analyze differences in microvessel density and size.

\section{Cells}

Human dermal lymphatic endothelial cells (LECs) were isolated from neonatal human foreskins by immunomagnetic purification as described. ${ }^{18}$ Lineage-specific differentiation was confirmed by means of real-time RT-PCR for the lymphatic vascular markers Prox1, LYVE-1, and podoplanin, and for the blood vascular endothelial markers VEGF receptor-1 and VEGF-C, as well as by immunostaining for CD31, Prox1, and podoplanin as described. ${ }^{19}$ Cells were cultured in endothelial basal medium (EBM) 2 (Lonza, Basel, Switzerland) with supplements, for up to 11 passages.

\section{Immunoblotting and Quantitative Real-Time $R T-P C R$}

Western blot analyses of Tie1, Tie2, ZO-1, and claudin-5 were performed as described. ${ }^{19}$ Briefly, confluent LECs were homogenized in lysis buffer, and protein concentrations were determined using the BCA-Kit (Pierce Biotech- 
nology, Rockford, IL). LECs also were treated with 500 $\mathrm{ng} / \mathrm{mL}$ Ang 1 for 4 hours for the detection of claudin- 5 and ZO-1, or for 10 minutes for the detection of phosphorylated Tie2 and Tie2. Equal amounts of lysates (100 $\mu \mathrm{g}$ protein) were immunoprecipitated with a rabbit polyclonal antibody against Tie1 or Tie2 (Santa Cruz Biotechnology) and then immunoblotted with a rabbit polyclonal antibody against Tie1, Tie2 (Santa Cruz Biotechnology), or phospho-Tie2 (Cell Signaling, Danvers, MA). In other experiments, equal amounts of lysates (5 $\mu \mathrm{g}$ protein) were immunoblotted with rabbit polyclonal antibody against Tie1, Tie2, claudin-5 (Santa Cruz Biotechnology), or ZO-1 (Invitrogen). Specific binding was detected by means of the enhanced chemiluminescence system (Amersham Biosciences, Piscataway, NJ). Equal loading was confirmed with an antibody against $\beta$-actin (Sigma, St. Louis, MO). In addition, total RNA was isolated from LECs cultured in the presence or absence of Ang1 (10 to 100 ng) for 4 hours after serum starvation. The expression of claudin- 5 mRNA was examined by quantitative real-time RT-PCR, using the ABI Prism 7000 Sequence Detection System (Applied Biosystems, Foster City, CA). The probes and primers for claudin-5 and Tie2 were predesigned by Applied Biosystems (assay IDs: Hs00533949_m1 and Hs00176096_m1, respectively). Expression levels were normalized with respect to $\beta$-actin as an internal control (forward primer: 5'-TCACCGAGCGCGGCT-3', reverse primer: 5'-TAATGTCACGCACGATTTCCC-3') and probe (5'-FAM-CAGCTTCACCACCACGGCCGAG -TAMRA-3').

\section{siRNA Transfection}

Small interfering RNA (siRNA) transfection was performed using the Basic Nucleofactor Kit for primary mammalian endothelial cells (Amaxa Biosystems, Cologne, Germany) as described. ${ }^{20}$ Briefly, after trypsinization, LECs $\left(5 \times 10^{5}\right)$ were resuspended in $100 \mu \mathrm{L}$ of basic nucleofactor solution. Cells were transfected by electroporation (Nucleofactor II; Amaxa Biosystems), using 2.0 $\mu \mathrm{g}$ siRNA containing two different double-stranded oligonucleotides for Tie1, Tie2, claudin-5, or control siRNA. The following siRNAs were used: Tie2: 5'-GGUGCCAUGGACUUGAUCUdTdT-3' and 5'-GGCUAGUAAGAUCAAUGGUdTdT-3', Tie1: 5'-GGUGACACCGCUGUACUUUdTdT-3' and 5'-GGUUACUUGUAUAUCGCUAdTdT-3', claudin-5: 5'GGCUAAGAAUCUGCUUAGUdTdT-3' and 5'-CGGAAUGAAGUUUCCUUUU-3'. Control siRNA (silencer negative control \#1 siRNA; Ambion, Cambridgeshire, UK) comprised a 19-bp scrambled sequence with $3^{\prime}$ dT overhangs, having no significant sequence homology to any known gene sequence. At 72 hours after transfection, cells were used for immunoblotting or assays. Efficient knockdown of these genes was confirmed by immunoblotting.

\section{Migration, Cord Formation, and Permeability Assays}

Haptotactic cell migration of LECs was studied as described, ${ }^{19}$ using 24-well FluoroBlok inserts of $8-\mu \mathrm{m}$ pore size (Falcon, Franklin Lakes, $\mathrm{NJ}$ ). Briefly, the bottom sides of the inserts were coated with $10 \mu \mathrm{g} / \mathrm{mL}$ fibronec-

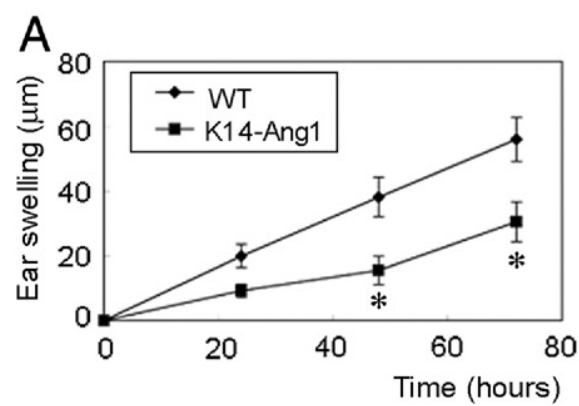

Figure 1. K14-Ang1 mice showed attenuated edema formation and inflammation induced by UVB. A: UVB exposure of WT mice resulted in a gradual increase of ear thickness (diamonds) with a maximum at 72 hours after the irradiation; however, the extent of ear swelling was decreased significantly in K14-Ang1 mice (squares) at 48 and 72 hours after UVB, as compared with WT mice $(N=5$ for each group). B-I: H\&E and CD11b stainings revealed attenuation of edema formation and macrophage infiltration in the dermis of K14-Ang1 mice after UVB ( $\mathbf{E}$ and $\mathbf{I}$ ) as compared with WT mice (D and $\mathbf{H}$ ), whereas no difference was apparent between WT (B and F) and K14-Ang1 (C and G) mice without UVB exposure. ${ }^{*} P<0.01$. Scale bars $=100 \mu \mathrm{m}$.

WT

B

HE
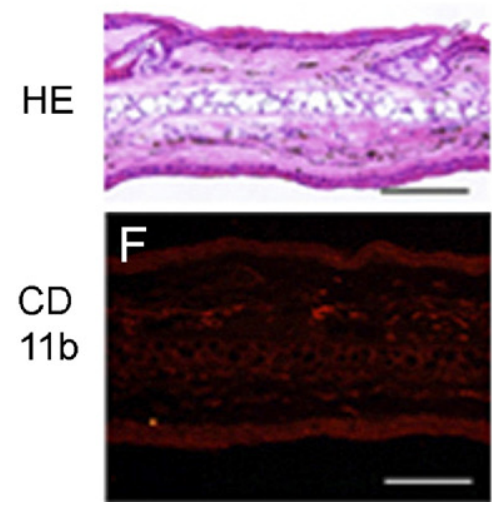

K14-Ang1

C
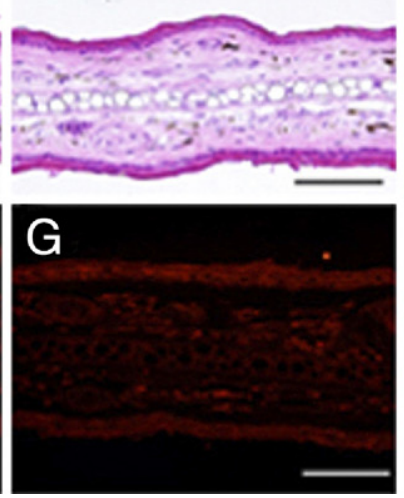

UVB
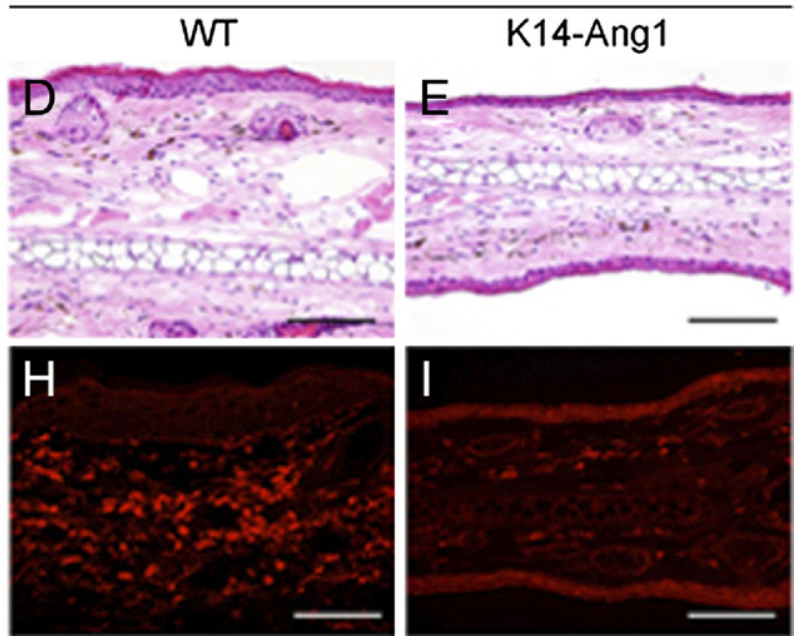
A

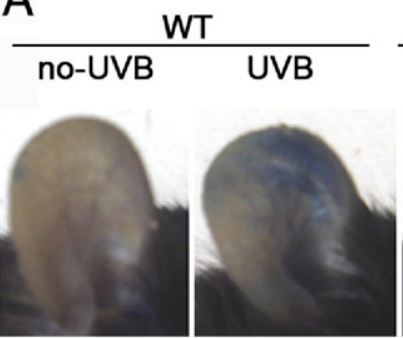

$\frac{\text { K14-Ang1 }}{\text { no-UVB UVB }}$

B

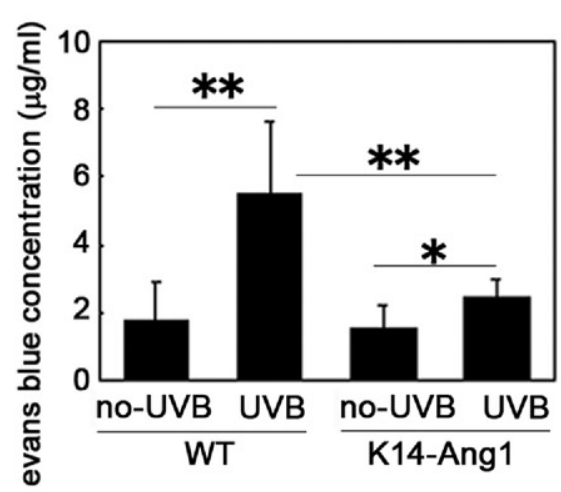

C

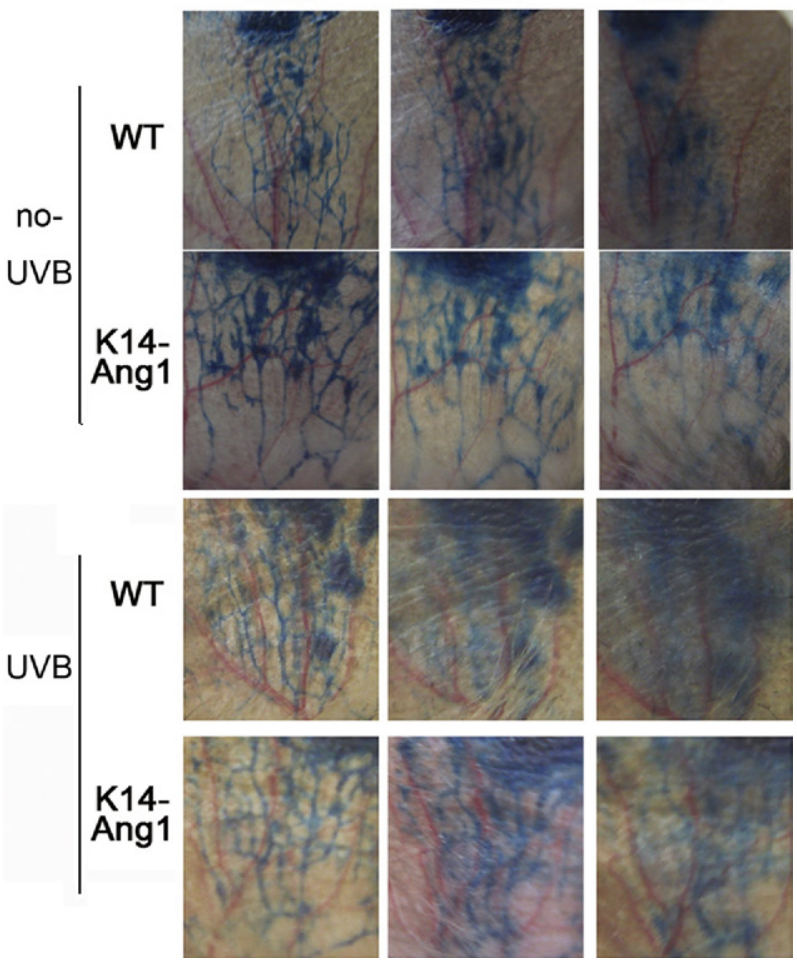

Figure 2. Decreased hyperpermeability of blood vessels as well as lymphatic vessels in K14-Ang1 mice during inflammation. A: The Miles assay revealed that UVB exposure induced vascular hyperpermeability of WT mice, whereas this effect was inhibited markedly in K14-Ang1 mice, as compared with WT. B: Quantitative analysis showed increased Evans blue leakage in the ear of UVB-irradiated WT mice as compared with UVBirradiated K14-Ang1 mice ( $N=3$ for each group). C: Intravital lymphangiography revealed that lymph leakage was apparent in the whole ear of UVB-exposed WT mice at 5 and 10 minutes after dye injection, whereas the leakage was attenuated markedly in K14-Ang1 mice as compared with WT. Without UVB, there was no significant difference of lymph leakage at 5 minutes, however, at 10 minutes K14-Ang1 mice showed inhibited dye leakage. ${ }^{*} P<0.05,{ }^{* *} P<0.01$.
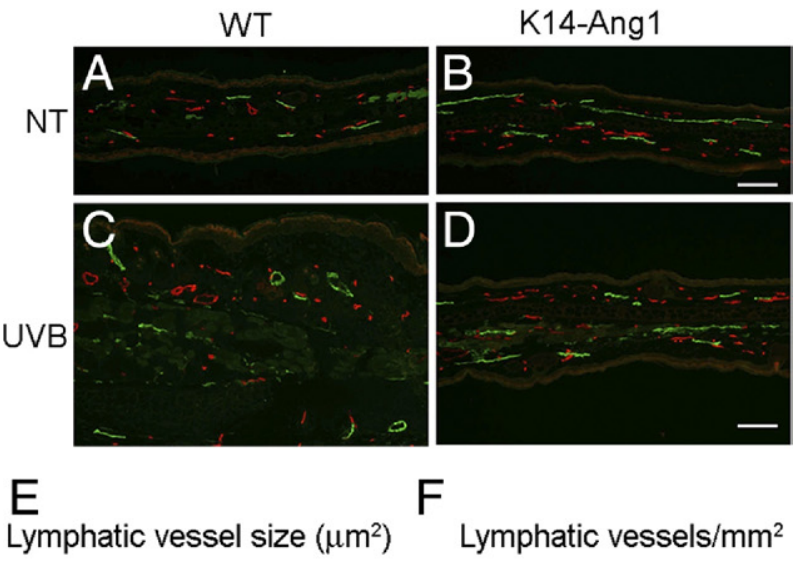

$\mathrm{F}$

Lymphatic vessel size $\left(\mu \mathrm{m}^{2}\right)$

Lymphatic vessels $/ \mathrm{mm}^{2}$
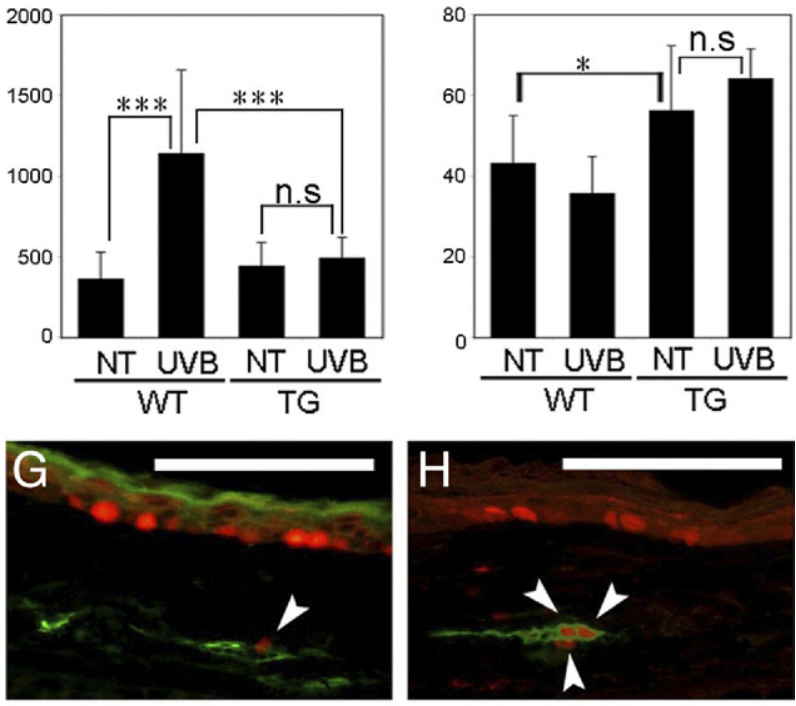

I

Blood vessel size $\left(\mu \mathrm{m}^{2}\right)$

$J$
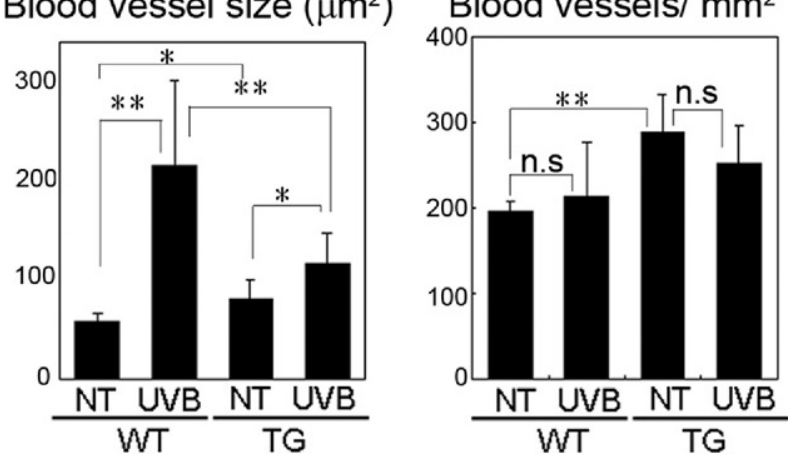

Figure 3. Inhibited enlargement of lymphatic vessels in inflamed K14-Ang1 mice. A-D: Double-immunofluorescence analysis using antibodies for blood vessel-specific antigen, panendothelial antigen-1 (red), and LYVE-1 (green) revealed marked enlargement of LYVE-1-positive lymphatic vessels in UVB-exposed WT mice $(\mathbf{C})$ as compared with ear skin not exposed to UVB (non-UV) (A), whereas in K14-Ang1 mice, the enlargement of lymphatic vessels after UVB exposure was attenuated (D). In contrast, more lymphatic vessels were found in K14-Ang1 mice (B), as compared with WT mice (D). E and F: Morphometric analysis using ear sections stained with LYVE-1 showed pronounced enlargement of lymphatic vessels of WT mice after UVB irradiation, whereas the lymphatic enlargement was strongly inhibited in K14 Ang1 mice (E). The density of lymphatic vessels was increased in K14-Ang1 mice, as compared with WT mice (F). G and $\mathbf{H}$ : Double-immunofluorescence analysis for proliferation marker Ki-67 (red) and podoplanin (green) showed greater proliferation of lymphatic endothelial cells in K14-Ang1 mice (H, arrowheads) as compared with WT mice (G, arrowheads). I and J: Under physiological conditions, the size (I) and density (J) of blood vessels were increased in K14-Ang1 mice. After UVB irradiation, the change of blood vessel size was smaller in K14-Ang1 mice than in WT mice. The density was comparable in skins exposed and not exposed to UVB $(N=$ 5 for each group). ${ }^{*} P<0.05,{ }^{* * * *} P<0.01$, and ${ }^{* * * * *} P<0.001$. Scale bars $=100 \mu \mathrm{m}$. 
tin (BD Bioscience) for 1 hour. LECs (100 $\mu \mathrm{L} ; 1 \times 10^{6}$ cells $/ \mathrm{mL}$ ) in serum-free EBM were seeded into the upper chambers and incubated for 4 hours at $37^{\circ} \mathrm{C}$ in the presence of Ang1 (5 to $500 \mu \mathrm{g} / \mathrm{mL}$ ). The fluorescence intensity (proportional to the number of viable cells) was measured using a Fluoroskan Ascent (Thermo Fisher Scientific, Waltham, MA). Cord-formation assays were performed as described. ${ }^{19}$ LECs after transfection of either Tie1, Tie2, or control siRNA were grown on fibronectin-coated 24-well plates until confluence. Then, $0.5 \mathrm{~mL}$ of a neutralized isotonic bovine dermal collagen type I solution (Vitrogen, Palo Alto, CA) with or without Ang1 (500 $\mathrm{ng} / \mathrm{mL}$ ) was added to the cells. After incubation at $37^{\circ} \mathrm{C}$ for 6 hours, cells were fixed with $4 \%$ paraformaldehyde for 30 minutes at $4^{\circ} \mathrm{C}$. Representative images were cap- tured and the total length of tube-like structures per area was measured using the IP-LAB software, as described. ${ }^{19}$ A permeability assay also was performed. Briefly, LECs were grown to confluence on the fibronectin-coated surface of $0.4-\mu \mathrm{m}$ pore size tissue culture inserts (Corning, Lowell, MA), followed by culture in serumfree EBM for 24 hours. Then, the upper and lower chambers were cultured in the presence or absence of angiopoetin-1 (50 to $500 \mathrm{ng} / \mathrm{mL}$ ) together with S-nitroso$\mathrm{N}$-acetylpenicillamine ${ }^{2 \mathrm{O}}$ (Sigma) for 6 hours. fluorescein isothiocyanate-dextran was added to the upper chambers, and after incubation for 15 minutes the concentration of fluorescein isothiocyanate-dextran in the lower chambers was determined at $492 \mathrm{~nm}$ using a Fluoroskan Ascent spectrophotometer (Thermo Fisher Scientific).
A

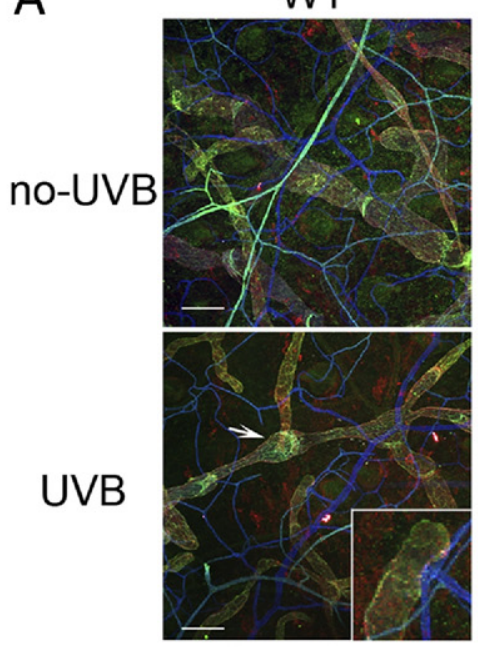

B
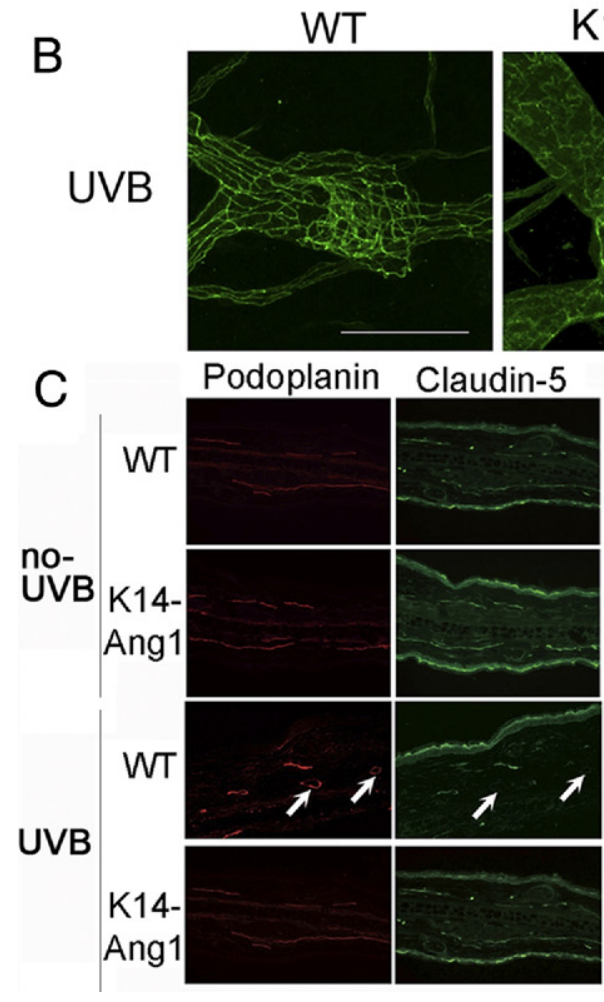

\section{K14-Ang1}
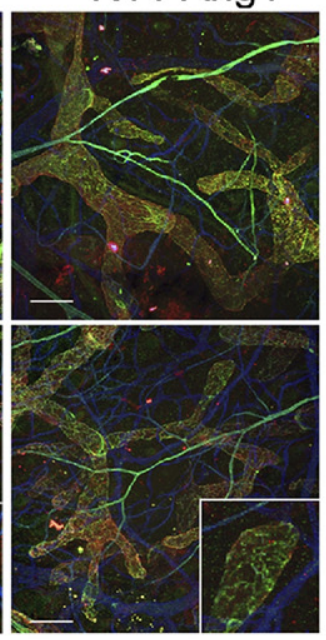

K14-Ang1

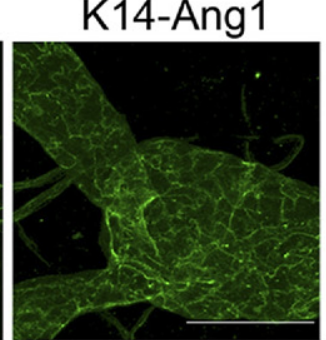

Merge
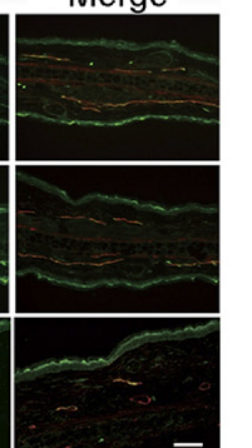

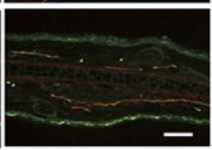
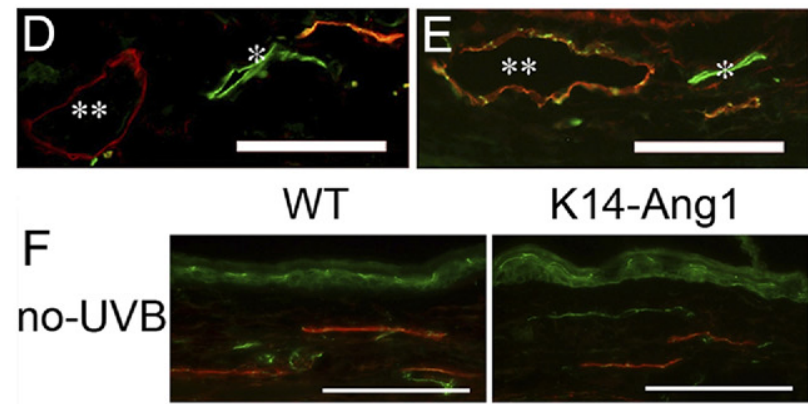

K14-Ang1

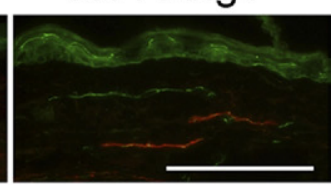

UVB
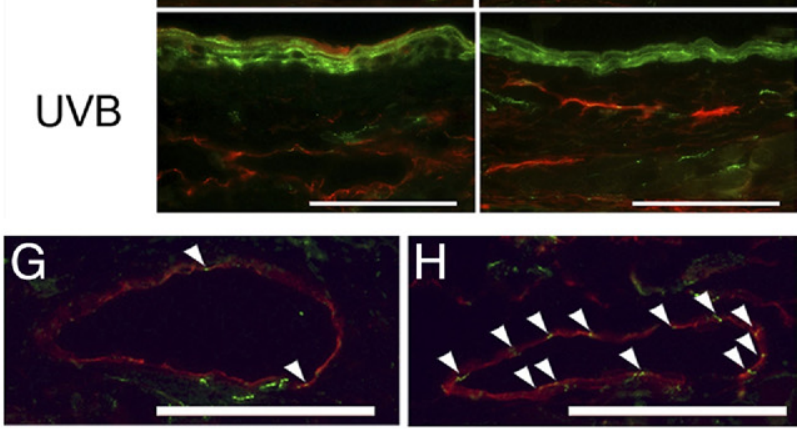

Figure 4. Lymphatic integrity is promoted in K14-Ang1 mice after UVB irradiation. A: Whole-mount staining of mouse ear using antibodies against CD31 (blue), podoplanin (red), and claudin-5 (green) revealed that UVB irradiation lead to the loss of claudin-5 protein, which was localized exclusively to the cell-cell junction at the tips of lymphatic capillaries without UVB, whereas claudin- 5 expression was already at the cell-cell junctions in UVB-irradiated K14-Ang1 mice. An arrow shows swollen collecting vessels. B: Confocal microscopy revealed that claudin -5 protein (green) was present in the cellular membrane of collecting lymphatic vessels in skin, whereas its expression was diminished in inflamed skin. C: Double-immunofluorescence analysis using antibodies against podoplanin (red) and claudin-5 (green) also confirmed that UVB irradiation resulted in the loss of claudin- 5 expression at cell-cell junctions (arrows). D and E: Higher magnification of podoplanin (red) and claudin-5 (green) staining. Loss of claudin-5 in lymphatics of UVB-irradiated WT mice indicated by double asterisks (D), already was redistributed in the cell-cell junctions of K14-Ang1 mice (E). Single asterisk indicates podoplanin-negative claudin-5-positive blood vessels $(\mathbf{F}-\mathbf{H})$. Double-immunofluorescence staining for podoplanin (red) and ZO-1 (green) shows ZO-1 expression in lymphatic vessels was lost in the UVB-irradiated skin of WT mice (high magnification with arrowheads in G), and the level was markedly less than that in UVB-exposed K14-Ang1 mice (high magnification with arrowheads in $\mathbf{H}$ ). Scale bars $=100 \mu \mathrm{m}$. 
Claudin-5-siRNA transfected or control-siRNA transfected LECs also were cultured on the fibronectin-coated surface of 0.4- $\mu \mathrm{m}$ pore size tissue culture inserts for 2 days, followed by incubation in serum-free EBM for 24 hours. Then, fluorescein isothiocyanate was added to the upper chambers and the concentration of fluorescein isothiocyanate in the lower chambers was measured. All studies were performed in triplicate. Statistical analyses were performed using the unpaired Student's t-test.

\section{Results}

\section{Edema Formation and Inflammation Induced by} Acute UVB Irradiation Are Attenuated in

\section{K14-Ang1 Mice}

To determine the role of Ang1 in cutaneous inflammation, K14-Ang1 mice and WT mice were exposed to $200 \mathrm{~mJ} /$ $\mathrm{cm}^{2}$ of UVB irradiation. Pronounced ear swelling was observed in WT mice, starting at day 2 after UVB irradiation; however, the ear swelling was attenuated significantly in K14-Ang1 mice (Figure 1A). Histologic analysis revealed no major physiological difference between WT mice and K14-Ang1 mice in the absence of UVB exposure (Figure 1, B and C). The ears of UVB-irradiated WT mice showed the characteristics of inflammation, such as epidermal hyperplasia and dermal edema, whereas these changes were ameliorated in K14-Ang1 mice (Figure 1, D and E). Immunohistochemical analysis of a monocyte-macrophage marker, CD11b, indicated marked macrophage infiltration in UVB-exposed WT mouse ear, whereas the number of infiltrated macrophages was decreased in K14-Ang1 mice (Figure 1, H and I). Few or no CD11b-positive macrophages were found in WT or K14-Ang1 mice not exposed to UVB (Figure 1, F and G).

\section{Promotion of Lymphatic and Blood Vascular Function of K14-Ang1 Mice during Inflammation}

First, a Miles assay was performed to determine the effects of Ang1 on blood vessels. UVB exposure induced marked leakage of Evans blue dye in WT mice, whereas K14-Ang1 mice showed decreased leakiness, as compared with WT mice (Figure 2A). Quantitative analysis showed that UVB irradiation strongly enhanced dye leakage in WT mouse ear, whereas the increase of dye leakage was inhibited markedly in UVB-irradiated K14-Ang1 mice, although there was still a significant difference between nonirradiated and UVB-irradiated K14-Ang1 mice

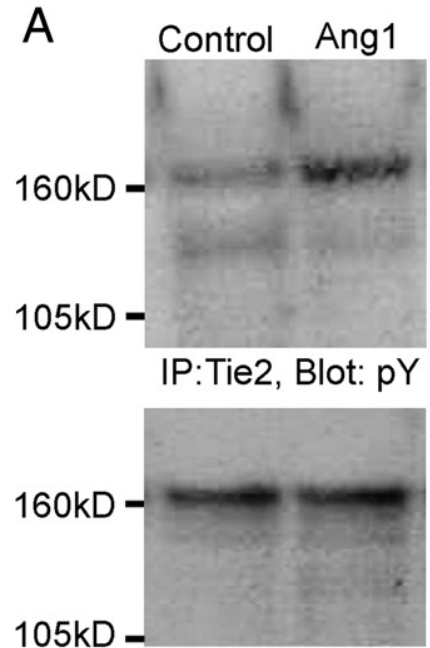

IP:Tie2, Blot: Tie2
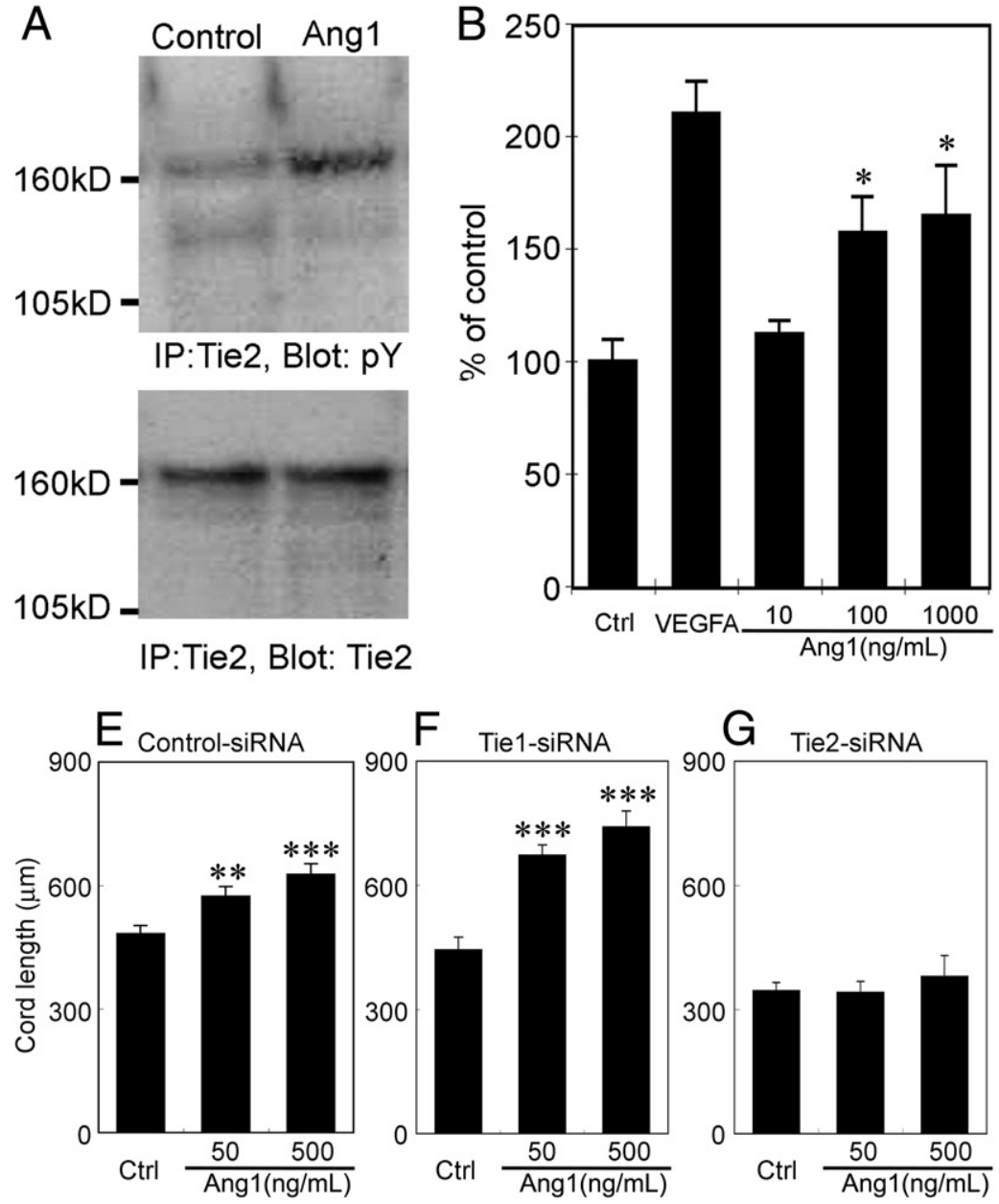

C

$\begin{array}{llr}\text { Control- } & \text { Tie1- } & \text { Tie2- } \\ \text { siRNA } & \text { siRNA } & \text { siRNA }\end{array}$

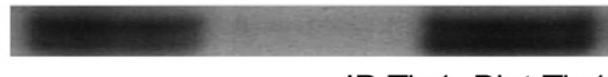

IP:Tie1, Blot:Tie1
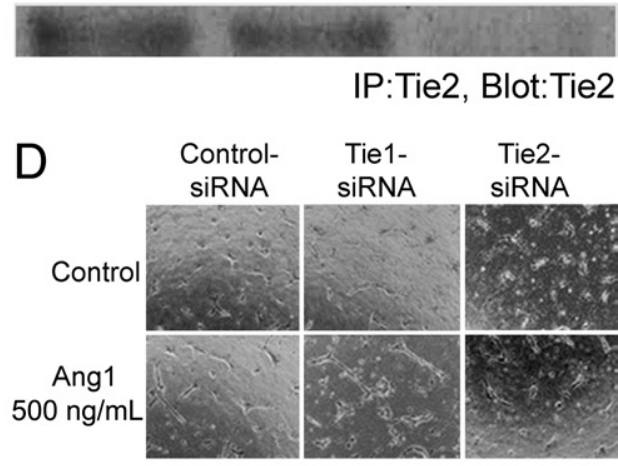

Figure 5. Ang1 promotes migration and cord-like structure formation of LECs via activation of Tie2 signaling. A: Treatment of LECs with Ang1 resulted in phosphorylation of Tie2 protein. Immunoblotting of Tie 2 confirmed equal protein loading. B: Ang1 dose-dependently induced migration of LECs. C: Immunoblot analysis for Tie1 or Tie 2 confirmed knockdown of Tie 1 or Tie2 protein by transfection of the respective siRNAs. D Representative pictures of cord-like structure formation induced by Ang1. Ang1 promoted cord formation of control-siRNA/Tie1siRNA-transfected LECs, whereas Tie2-siRNA transfection of LECs diminished the Ang1-induced cord formation. E-G: Morphometric analysis confirmed the dose-dependent induction of cord formation in control-siRNA-transfected (E) and Tie1siRNA-transfected (F) LECs by Ang1, whereas no effect of Ang1 was seen in Tie2-siRNA-transfected (G) LECs. At least three different experiments were performed for each assay to calculate $P$ values. IP, immunoprecipitation. ${ }^{*} P<0.05$, ${ }^{* * *} P<0.01,{ }^{* * * *} P<0.001$. 
(Figure 2B). Next, to visualize lymphatic function, Evans blue dye was injected intradermally into mouse ear. Lymph leakage was found in the whole ear of UVB-irradiated WT mice at 5 and 10 minutes after dye injection as previously described, ${ }^{8}$ whereas lymphatic vessels of UVB-exposed K14-Ang1 mice were still visible at 5 and 10 minutes after dye injection, indicating that the increase of lymphatic capillary permeability was attenuated in K14-Ang1 mice. Moreover, at 1 minute after dye injection, dye spots were found close to the branching points of lymphatic vessels only in UVB-irradiated WT mice, suggesting the presence of abnormal and leaky collecting vessels (Figure $2 \mathrm{C}$ ). In contrast, in non-UV, there was no significant difference between WT mice and K14-Ang1 in terms of lymph leakage at 5 minutes after dye injection, however, at 10 minutes, K14-Ang1 mouse ears showed inhibited lymph leakage as compared with WT mice.

Double-immunofluorescence analysis using antibodies for blood vessel-specific antigen, panendothelial antigen- $1,{ }^{21}$ and a lymphatic specific marker, LYVE-1, was performed. UVB induced marked enlargement of LYVE1-positive lymphatic vessels in WT mice as compared with ear skin not exposed to UVB (Figure 3, A and C), whereas in K14-Ang1 mice, the enlargement of lymphatic vessels and blood vessels after UVB was attenuated, as compared with WT. In contrast, the density of lymphatic vessels was increased in K14-Ang1 mice compared with WT mice (Figure 3, B and D). Morphometric analysis of lymphatic vessels using IP-LAB software showed an increase in the average size of the inflamed lymphatic vessels of WT mice as compared with the vessels of nonirradiated WT mice, although no significant difference was found in the vessel density. As we had expected, the average size of lymphatic vessels was decreased in K14Ang1 mice after UVB irradiation. In contrast, the density of lymphatic vessels was increased in K14-Ang1 mice, as compared with WT mice (Figure 3, E and F). To analyze if the increased area of lymphatic vessels in K14-Ang1 mice resulted from the presence of more lymphatic endothelial cells, double-immunofluorescence analysis for podoplanin and a proliferation marker, Ki-67, was performed. The results showed that the number of Ki-67positive cells was increased in K14-Ang1 mice as compared with WT mice $(1.75 \pm 0.96$ cells/slide in K14-Ang 1 mice and $0.25 \pm 0.5$ cells/slide in WT mice; $P=0.016$ ) (Figure 3, G and $\mathrm{H}$ ). Under physiological conditions, the size and density of blood vessels were increased in K14Ang1 mice, as previously described (Figure $3, A$ and B). ${ }^{14}$ After UVB irradiation, a relatively small change of blood vessel size was found in K14-Ang1 mice as compared with WT mice. The vessel density was comparable in skin exposed and not exposed to UVB (Figure 3, I and J).

\section{Enhanced Lymphatic Integrity in Inflamed Ears of K14-Ang1 Mice}

We previously found that the enlarged lymphatic vessels induced by UVB irradiation are leaky and hyperpermeable, suggesting that lymphatic function is impaired. ${ }^{8}$ Therefore, we hypothesized that altered expression pat- tern of tight junction molecules might be associated with the leakiness of inflamed lymphatic vessels. To test this idea, we performed whole-mount staining for claudin-5 and/or podoplanin/CD31 in ears of animals exposed or not exposed to UVB irradiation. Interestingly, UVB irradiation led to the loss of claudin-5 protein, which was localized exclusively to cell-cell junctions at the tips of lymphatic capillaries without UVB, whereas claudin-5 expression already was redistributed at cell-cell junctions in UVB-irradiated K14-Ang1 mice (Figure 4A). Confocal

A
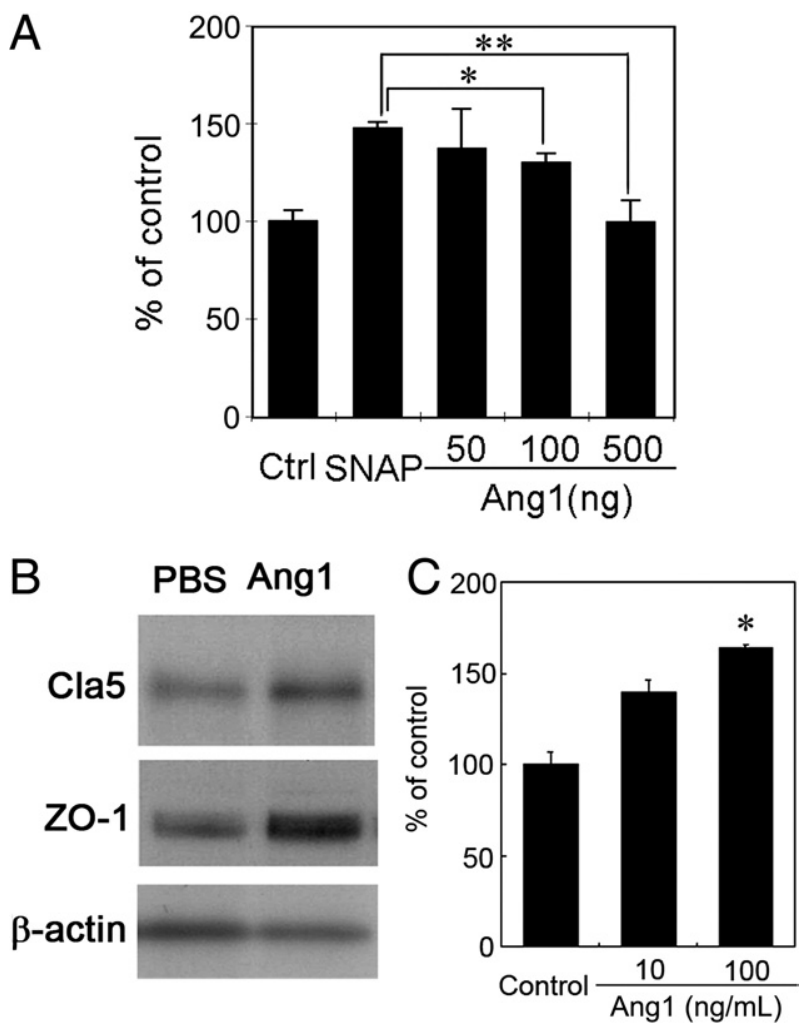

D Tie2 expression
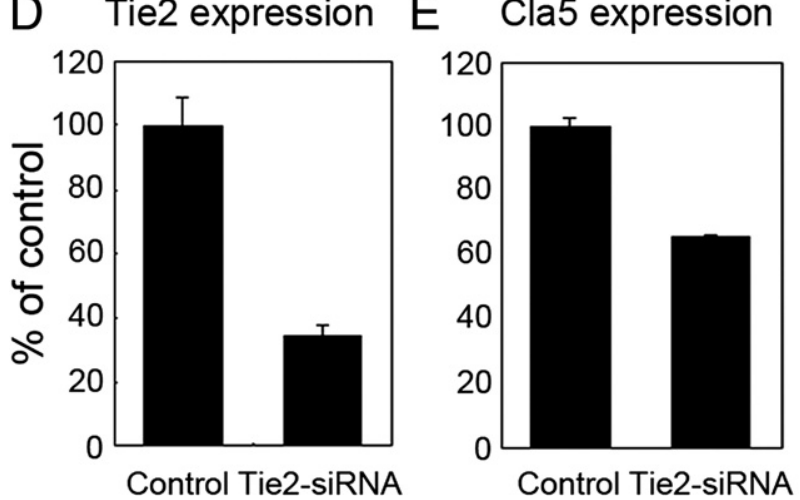

Figure 6. Ang1 promotes the integrity of LECs by increasing expression of tight junction molecules. A: Nitric oxide donor S-nitroso-N-acetylpenicillamine (SNAP) increased the lymphatic permeability, whereas co-incubation of LECs with Ang1 and SNAP dose-dependently inhibited the nitric oxideinduced hyperpermeability of LECs. B: Treatment with Ang1 for 4 hours markedly increased the levels of tight junction molecules claudin- 5 and ZO-1. $\beta$-Actin is shown as a loading control. C: Claudin- 5 expression was dosedependently increased in the presence of Ang1. (D and E) LECs transfected with Tie2-siRNA showed decreased expression of Tie2 (D) and claudin-5 (E). At least three different experiments were performed for each assay to calculate $P$ values. ${ }^{*} P<0.05,{ }^{* *} P<0.01$. 
microscopy revealed that claudin-5 protein was present in the cellular membrane of collecting lymphatic vessels in skin, whereas its expression was diminished in inflamed skin (Figure 4B). These data suggested that the lymphatic capillaries and collecting vessels became leaky after UVB irradiation because of loss of claudin-5.

Next, to determine the expression pattern of the lymphatics in K14-Ang1 mice, immunofluorescence analyses using antibodies for the endothelial tight junction molecule claudin-5 and lymphatic vessels were performed. Double-immunofluorescence analysis using antibodies against podoplanin and claudin-5 also confirmed that UVB irradiation resulted in the loss of claudin-5 expression at cellular membranes of lymphatic vessels in WT mice. Moreover, loss of claudin-5 in lymphatics of UVB-irradiated WT mice (Figure 4D), already was redistributed in cell-cell junctions of K14-Ang1 mice (Figure 4E). Another tight junction molecule, ZO-1, also was expressed in podoplanin-positive lymphatic vessels. Double-immunofluorescence staining for podoplanin and ZO-1 showed that ZO-1 expression in lymphatic vessels was lost in the UVB-irradiated skin of WT mice, and the level was markedly less than that in UVB-exposed K14Ang1 mice. These data indicate that the lymphatic integrity of inflamed skin of UVB-exposed K14-Ang1 mice was enhanced, as compared with WT.

\section{Tie2 Activation by Ang1 Induces Cell Migration and Cord Formation in Vitro}

To examine the role of Tie2 signaling in LECs, immunoblot analysis was performed after immunoprecipitation of Tie2. In the presence of Ang1, pronounced Tie2 phosphorylation was found as compared with the control cells, although immunoblot analysis of Tie2 protein confirmed equal loading of protein (Figure 5A). Next, we investigated the effect of Ang1 on LECs. Treatment of LECs with Ang1 dose-dependently induced migration (Figure 5B). The effect of VEGF-A also was assessed as a positive control. Further, Ang1 dose-dependently promoted cord formation of LECs. Our results are consistent with a previous finding of Tie1 phosphorylation of LECs in the presence of Ang1.

To determine which pathway is critical in the mediation of Ang1 signaling of LECs, the Tie2 or Tie1 receptor was specifically knocked down by the transfection of silencer RNAs. We confirmed specific knockdown of Tie1 or Tie2 expression by immunoblotting (Figure 5C). In addition, a cord-formation assay of these cells was performed. Ang1 promoted cord formation of Tie1-knockdown cells as well as control siRNA-transfected cells, whereas in cells transfected with siRNA of Tie2, the effect of Ang1 was reduced, showing the importance of Ang1/Tie2 signaling in LECs (Figure 5, D-G).

\section{Ang1 Enhances Lymphatic Integrity by Increasing Levels of Tight Junction Molecules in Vitro}

In vivo, K14-Ang1 mice showed increased levels of tight junction molecules in inflamed skin. To analyze the contribution of Ang1/Tie2 signaling to lymphatic integrity, a Transwell permeability assay was performed in the presence or absence of Ang1 together with a nitric oxide donor, S-nitroso-N-acetylpenicillamine. ${ }^{20}$ Ang1 dose-dependently inhibited the lymphatic permeability (Figure $6 A$ ). Furthermore, we detected increased expression of claudin-5 as well as ZO-1 after the addition of Ang1, whereas $\beta$-actin expression was similar in the two groups (Figure 6B). Moreover, Ang1 treatment of LECs dosedependently enhanced the expression of claudin-5 (Figure 6C), suggesting that Ang1 induces an increase of lymphatic integrity by promoting expression of tight junction molecules. In contrast, the transfection of LECs with siRNA-Tie2 decreased claudin-5 expression (Figure 6, D and $\mathrm{E}$ ). These data indicate that Ang1/Tie2 signaling influences lymphatic permeability by modulating expression of tight junction molecules.
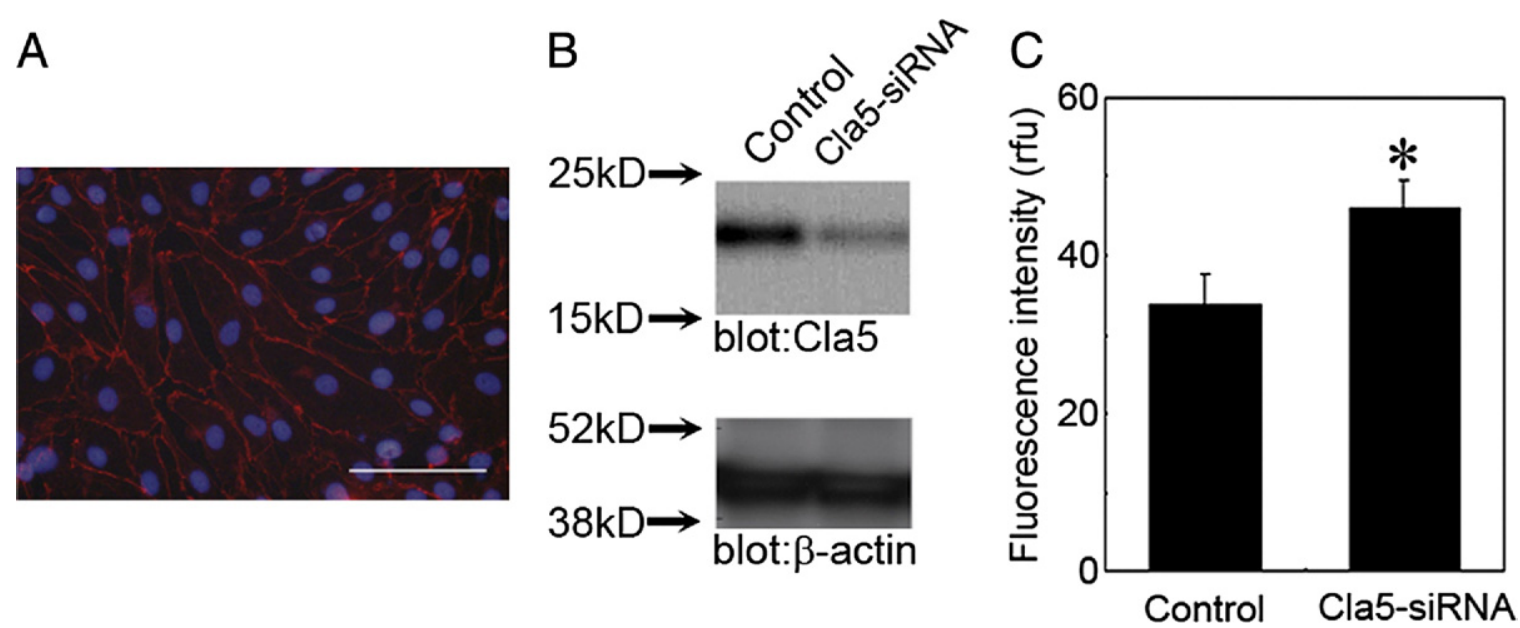

Figure 7. Claudin-5 regulates lymphatic integrity in vitro. A: Immunofluorescence of LECs for claudin-5 (red) confirmed the expression of claudin-5 on the cellular membrane of LECs. B: Western blot analyses revealed a significant decrease of claudin-5 protein in the presence of claudin-5-specific siRNA, whereas $\beta$-actin protein levels were comparable in LECs transfected with scramble-siRNA and with claudin- 5 siRNA. C: Claudin- 5 siRNA transfection resulted in increased permeability of LECs in vitro. Three different experiments were performed for each assay. ${ }^{*} P<0.001$. Scale bar $=100 \mu \mathrm{m}$. 


\section{Claudin-5 Influences Lymphatic Permeability}

Claudin-5 expression was increased in the cellular membrane of lymphatic endothelial cells in vitro (Figure 7A). To elucidate claudin-5 function in LECs, claudin-5 expression was knocked down with claudin- 5 siRNA; $\beta$-actin expression remained similar in the control and claudin-5 siRNA transfectants (Figure 7B). Claudin-5 knockdown resulted in increased permeability of LECs in vitro (Figure 7C).

\section{Discussion}

Complex phenomena occur during skin inflammation, including epidermal hyperplasia, erythema, edema formation, vessel dilation, and infiltration of inflammatory cells. ${ }^{1}$ It also has been shown that lymphatic function actively participates in the resolution of inflammation by modulating lymphangiogenic factors, VEGF-A and VEGF-C/D, secreted from macrophages. ${ }^{4}$ In our skin inflammation model, the subcutaneous delivery of VEGF-C attenuated skin inflammation by promoting lymphangiogenesis. ${ }^{6}$ In contrast, VEGF-A up-regulation in keratinocytes triggered lymphatic impairment, whereas systemic blockade of VEGF-A attenuated skin inflammation by inhibiting the enlargement of lymphatic vessels. ${ }^{8}$ These data indicate that lymphangiogenic factors could play distinct roles in inflammation resolution.

Our results showed that activation of Ang1/Tie2 signaling attenuated inflammation by promoting lymphatic integrity, as well as inhibiting blood vascular hyperpermeability in inflamed tissue. Blood vessels of K14-Ang1 mice were shown to be resistant to leakage induced by an inflammatory stimulus. ${ }^{22}$ Therefore, the effect of Ang1 on blood vessels may contribute to the attenuation of inflammation in K14-Ang1 mice. Recently, Ang1 was shown to promote lymphatic formation and hyperplasia. ${ }^{9,10} \mathrm{We}$ also confirmed increased lymphatic density in the skin of K14-Ang1 mice as compared with WT mice under physiological conditions. Interestingly, the enlargement of lymphatic vessels in inflamed skin of UVB-exposed K14Ang1 mice was reduced, compared with UVB-exposed WT mice. Furthermore, intravital lymphangiography by injection of Evans blue dye into the ear of UVB-exposed K14-Ang1 mice indicated that the increase of lymphatic permeability also is reduced in these mice. Unlike VEGF-C, Ang1 had no effect on proliferation of lymphatic endothelial cells in vitro (data not shown), although Ang1 dose-dependently promoted cell migration and cord formation. Taken together, these results indicate that, in addition to the well-known effect of Ang1 on blood vessels in inflammation, ${ }^{23}$ Ang1 has a distinctive functional role in lymphatic vessels of inflamed tissue, serving to modulate lymphatic integrity.

How is lymphatic integrity regulated in inflammation? Lymphatic hyperplasia, together with lymphatic enlargement, is found in several models of inflammation. 3,12 Surprisingly, loss of the tight junction molecule claudin-5 was found in inflamed lymphatic capillaries, as well as collecting vessels. In inflamed skin, lymphatic vessels could be pulled open by anchoring filaments that connect lymphatic endothelial cells with elastic fibers in the extracellular matrix, presumably to wash out increased interstitial tissue fluid resulting from the increased vascular permeability. ${ }^{24}$ However, overextension of lymphatic endothelial cells also can cause edema formation. ${ }^{25}$ Our results showed that overextension of lymphatic endothelial cells in capillaries as well as collecting vessels was caused by loss of tight junction molecules, claudin-5 and ZO-1 at cell-cell junctions, resulting in lymphatic impairment and prolonged edema and inflammation.

Recent results have indicated that VEGF-A induces disruption of claudin-5 in the blood-brain barrier. ${ }^{26}$ Further, up-regulation of VEGF-A was detected in keratinocytes after UVB exposure in vitro as well as in vivo, and VEGF-A was identified as a mediator of skin inflammation after UVB exposure. ${ }^{15,27}$ It would be of interest to see if VEGF-A up-regulation after UVB also mediates decreased expression of tight junction proteins in lymphatic vessels, followed by lymphatic impairment in inflamed skin. Furthermore, we found that activation of Ang1/Tie2 signaling increased the TJPs in vivo and in vitro. Adrenomedullin has been shown to control murine lymphatic development by stabilizing the lymphatic endothelial barrier, ${ }^{28,29}$ and its receptor-modulator, receptor activity modifying protein-2 (RAMP2), modulated vascular integrity in mice. ${ }^{30}$ We also confirmed that an endothelialspecific TJP, claudin-5, is a key modulator of the lymphatic endothelial barrier in vitro. However, in contrast to adrenomedullin, no obvious change of TJPs was found in K14-Ang1 mice under physiological conditions in vivo, whereas inflamed lymphatic vessels showed TJPs at cell-cell junctions in vivo, suggesting a distinct role of Ang1/Tie2 signaling in maintaining the lymphatic integrity of inflamed tissue.

In conclusion, we have identified a regulatory pathway that serves to maintain lymphatic integrity during inflammation by controlling TJP components. Ang1, or small molecules that directly activate Tie2, may have potential for the treatment of lymphatic dysfunction during inflammation.

\section{Acknowledgments}

We thank Koji Aruga, Fumika Miyohashi, and Junko Yamane for their technical assistance.

\section{References}

1. Cueni LN, Detmar M: New insights into the molecular control of the lymphatic vascular system and its role in disease. J Invest Dermatol 2006, 126:2167-2177

2. Tammela T, Alitalo K: Lymphangiogenesis: molecular mechanisms and future promise. Cell 2010, 140:460-476

3. Baluk P, Tammela T, Ator E, Lyubynska N, Achen MG, Hicklin DJ, Jeltsch M, Petrova TV, Pytowski B, Stacker SA, Yla-Herttuala S, Jackson DG, Alitalo K, McDonald DM: Pathogenesis of persistent lymphatic vessel hyperplasia in chronic airway inflammation. J Clin Invest 2005, 115:247-257

4. Kataru RP, Jung K, Jang C, Yang H, Schwendener RA, Baik JE, Han $\mathrm{SH}$, Alitalo K, Koh GY: Critical role of CD11b+ macrophages and 
VEGF in inflammatory lymphangiogenesis, antigen clearance, and inflammation resolution. Blood 2009, 113:5650-5659

5. Alitalo K, Tammela T, Petrova TV: Lymphangiogenesis in development and human disease. Nature 2005, 438:946-953

6. Kajiya K, Sawane M, Huggenberger R, Detmar M: Activation of the VEGFR-3 pathway by VEGF-C attenuates UVB-induced edema formation and skin inflammation by promoting lymphangiogenesis. J Invest Dermatol 2009, 129:1292-1298

7. Nagy JA, Vasile E, Feng D, Sundberg C, Brown LF, Detmar MJ, Lawitts JA, Benjamin L, Tan X, Manseau EJ, Dvorak AM, Dvorak HF: Vascular permeability factor/vascular endothelial growth factor induces lymphangiogenesis as well as angiogenesis. J Exp Med 2002, 196:1497-1506

8. Kajiya K, Hirakawa S, Detmar M: Vascular endothelial growth factor-a mediates ultraviolet B-induced impairment of lymphatic vessel function. Am J Pathol 2006, 169:1496-1503

9. Morisada T, Oike Y, Yamada Y, Urano T, Akao M, Kubota Y, Maekawa H, Kimura Y, Ohmura M, Miyamoto T, Nozawa S, Koh GY, Alitalo K, Suda T: Angiopoietin-1 promotes LYVE-1-positive lymphatic vessel formation. Blood 2005, 105:4649-4656

10. Tammela T, Saaristo A, Lohela M, Morisada T, Tornberg J, Norrmen C, Oike Y, Pajusola K, Thurston G, Suda T, Yla-Herttuala S, Alitalo K: Angiopoietin-1 promotes lymphatic sprouting and hyperplasia. Blood 2005, 105:4642-4648

11. Augustin HG, Koh GY, Thurston G, Alitalo K: Control of vascular morphogenesis and homeostasis through the angiopoietin-Tie system. Nat Rev Mol Cell Biol 2009, 10:165-177

12. Baluk P, Fuxe J, Hashizume H, Romano T, Lashnits E, Butz S, Vestweber D, Corada M, Molendini C, Dejana E, McDonald DM: Functionally specialized junctions between endothelial cells of lymphatic vessels. J Exp Med 2007, 204:2349-2362

13. Nitta T, Hata M, Gotoh S, Seo Y, Sasaki H, Hashimoto N, Furuse M, Tsukita S: Size-selective loosening of the blood-brain barrier in claudin-5-deficient mice. J Cell Biol 2003, 161:653-660

14. Suri C, McClain J, Thurston G, McDonald DM, Zhou H, Oldmixon EH, Sato TN, Yancopoulos GD: Increased vascularization in mice overexpressing angiopoietin-1. Science 1998, 282:468-471

15. Yano K, Kajiya K, Ishiwata M, Hong YK, Miyakawa T, Detmar M: Ultraviolet B-induced skin angiogenesis is associated with a switch in the balance of vascular endothelial growth factor and thrombospondin-1 expression. J Invest Dermatol 2004, 122:201-208

16. Kidoya $\mathrm{H}$, Naito $\mathrm{H}$, Takakura $\mathrm{N}$ : Apelin induces enlarged and nonleaky blood vessels for functional recovery from ischemia. Blood 2010, 115:3166-3174
17. Hirakawa S, Fujii S, Kajiya K, Yano K, Detmar M: Vascular endothelial growth factor promotes sensitivity to ultraviolet B-induced cutaneous photodamage. Blood 2005, 105:2392-2399

18. Hirakawa S, Hong YK, Harvey N, Schacht V, Matsuda K, Libermann $\mathrm{T}$, Detmar M: Identification of vascular lineage-specific genes by transcriptional profiling of isolated blood vascular and lymphatic endothelial cells. Am J Pathol 2003, 162:575-586

19. Kajiya K, Hirakawa S, Ma B, Drinnenberg I, Detmar M: Hepatocyte growth factor promotes lymphatic vessel formation and function. EMBO J 2005, 24:2885-2895

20. Kajiya K, Huggenberger R, Drinnenberg I, Ma B, Detmar M: Nitric oxide mediates lymphatic vessel activation via soluble guanylate cyclase alpha1beta1-impact on inflammation. FASEB J 2008, 22: 530-537

21. Liersch R, Nay F, Lu L, Detmar M: Induction of Iymphatic endothelial cell differentiation in embryoid bodies. Blood 2006, 107: $1214-1216$

22. Thurston G, Suri C, Smith K, McClain J, Sato TN, Yancopoulos GD, McDonald DM: Leakage-resistant blood vessels in mice transgenically overexpressing angiopoietin-1. Science 1999, 286:2511-2514

23. Fiedler U, Augustin HG: Angiopoietins: a link between angiogenesis and inflammation. Trends Immunol 2006, 27:552-558

24. Gerli R, Solito R, Weber E, Agliano M: Specific adhesion molecules bind anchoring filaments and endothelial cells in human skin initial lymphatics. Lymphology 2000, 33:148-157

25. Skobe M, Detmar M: Structure, function, and molecular control of the skin lymphatic system. J Investig Dermatol Symp Proc 2000, 5:14-19

26. Argaw AT, Gurfein BT, Zhang Y, Zameer A, John GR: VEGF-mediated disruption of endothelial CLN-5 promotes blood-brain barrier breakdown. Proc Natl Acad Sci U S A 2009, 106:1977-1982

27. Yano K, Kadoya K, Kajiya K, Hong YK, Detmar M: Ultraviolet B irradiation of human skin induces an angiogenic switch that is mediated by upregulation of vascular endothelial growth factor and by downregulation of thrombospondin-1. Br J Dermatol 2005, 152:115121

28. Dunworth WP, Fritz-Six KL, Caron KM: Adrenomedullin stabilizes the lymphatic endothelial barrier in vitro and in vivo. Peptides 2008, 29:2243-2249

29. Fritz-Six KL, Dunworth WP, Li M, Caron KM: Adrenomedullin signaling is necessary for murine lymphatic vascular development. J Clin Invest $2008,118: 40-50$

30. Ichikawa-Shindo $Y$, Sakurai T, Kamiyoshi A, Kawate H, linuma N, Yoshizawa T, Koyama T, Fukuchi J, limuro S, Moriyama N, Kawakami H, Murata T, Kangawa K, Nagai R, Shindo T: The GPCR modulator protein RAMP2 is essential for angiogenesis and vascular integrity. $\mathrm{J}$ Clin Invest 2008, 118:29-39 